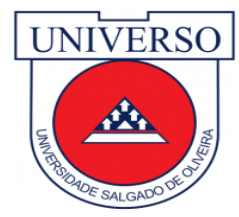

UNIVERSIDADE SALGADO DE OLIVEIRA

PRÓ-REITORIA ACADÊMICA

CURSO DE ENFERMAGEM

O ENFERMEIRO FRENTE À PREVENÇÃO DA TRANSMISSÃO VERTICAL DO HIV.

THALITA TAVARES RODRIGUES MENDONÇA

NITERÓI

2018 
THALITA TAVARES RODRIGUES MENDONÇA

\section{O ENFERMEIRO FRENTE À PREVENÇÃO DA TRANSMISSÃO VERTICAL DO HIV.}

Trabalho de conclusão de curso
apresentado à disciplina de TCC II Curso
de Enfermagem, da Universidade Salgado
de Oliveira - Universo como parte dos
requisitos para obtenção do título de
Bacharel em Enfermagem.
Orientador: Profa. Mestre Bruna Maiara
Ferreira Barreto Pires




\section{AGRADECIMENTOS}

A Deus, pois sem ele nada seria e não estaria aqui hoje. A minha família, por todo incentivo e apoio durante esses anos, vocês foram fundamentais nessa minha conquista. Aos meus mestres, por todo o conhecimento passado durante esses quatro anos, vocês foram de grande importância para que eu almejasse ser uma excelente profissional em um futuro muito próximo. A todos que me apoiaram e incentivaram a não desistir de lutar por meus sonhos e objetivos. 


\section{RESUMO}

A transmissão vertical é uma das principais vias de infecção do HIV, deve-se então dar mais importância para a prevenção dessa transmissão para que seja reduzido o número de crianças infectadas. No que se refere à assistência, o enfermeiro tem um papel muito importante no atendimento a essas mulheres bem como orientar a equipe de enfermagem para que todos os cuidados sejam realizados. A pesquisa tem como objetivo geral verificar a assistência do enfermeiro na prevenção da transmissão vertical do HIV. Notou-se uma necessidade da qualificação do enfermeiro para desempenhar uma assistência satisfatória e um apoio psicológico, pois o comprometimento psicológico da puérpera também dificulta a adesão ao tratamento. Há assim uma necessidade de conhecer a assistência de enfermagem prestada a essas puérperas, pois estudos apontam o aumento de crianças infectadas e o não comprometimento de muitas mulheres infectadas ao tratamento.

PALAVRAS-CHAVE: HIV; Assistência de enfermagem; Transmissão vertical. 


\begin{abstract}
The vertical transmission is one of the main ways of infection of the HIV, must then be given more importance for the prevention of this transmission so that the number of infected children is reduced. As for the assistance, the nurse has a very important paper in the attendance to these women as well as guiding the nursing team so that all the cares are carried through. The research has as objective generality to verify the assistance of nursing in the prevention of the transmission of the HIV. A necessity of the qualification of the nursing team was noticed to play a satisfactory assistance and a psychological support, therefore the psychological commitment of puerperal woman also makes it difficult the adhesion to the treatment. Postpartum women has thus a necessity to know the assistance of nursing given to these, therefore studies point the increase of infected children and not the commitment of many infected women to the treatment.
\end{abstract}

KEYWORDS: HIV; Assistance of nursing; Vertical transmission. 


\section{LISTA DE QUADROS}

Quadro 1. Título do artigo, autor (es), ano e objetivo principal - 15

Quadro 2. Título do artigo e principais resultados. - 16 
SUMÁRIO

1 INTRODUÇÃO 08

2 PROBLEMA 11

3 PRESSUPOSTOS 11

4 OBJETIVOS 11

4.1 OBJETIVO GERAL 11

4.2 OBJETIVOS ESPECÍFICOS

5 JUSTIFICATIVA E RELEVÂNCIA

6 MÉTODO 13

7 RESULTADO E DISCUSSÃO 14

7.1 HIV (VÍRUS DA IMUNODEFICIÊNCIA HUMANA) E A TRANSMISSÃO 16 VERTICAL

7.2 INCIDÊNCIAS DA TRANSMISSÃO VERTICAL 17

7.3 DIAGNÓSTICOS DA INFECÇÃO PELO HIV 18

7.4 MEDIDAS PROFILÁTICAS PARA PREVENÇÃO DO HIV 19

7.5 PAPEL DO ENFERMEIRO NA ASSISTÊNCIA A GESTANTE E A PREVENÇÃO 19

DA TRANSMISSÃO VERTICAL

8 CONCLUSÃO 22

9 REFERÊNCIAS 


\section{INTRODUÇÃO}

A AIDS (síndrome da imunodeficiência adquirida) é definida por uma disfunção grave do sistema imunológico, causada por um retrovírus denominado vírus da imunodeficiência humana (HIV) (LIMA et al, 2014).

O número de mulheres em idade reprodutiva HIV positivas está cada vez maior, o que propicia um maior risco de transmissão vertical do vírus. Este tipo de transmissão do vírus ocorre basicamente pela passagem do vírus da mãe para o bebê durante a gestação, o trabalho de parto, o parto ou durante a amamentação. Visando reduzir esse tipo de transmissão, o Ministério da Saúde preconiza uma série de medidas a serem adotadas durante o pré-natal, parto e puerpério, como por exemplo, a realização da testagem anti-HIV durante o pré-natal (SOARES et al, 2013).

Antigamente, quando surgiram os primeiros casos de AIDS em crianças, foi constatado que essa transmissão era de origem secundária, adquirida através de transfusão de sangue ou hemoderivados contaminados com o vírus HIV; a partir de 1985 surgiram os primeiros casos secundários à transmissão perinatal. No Brasil, no estado de São Paulo, em 1984, foi registrada a primeira ocorrência da transmissão perinatal, quando foram diagnosticados dois pacientes (TEIXEIRA et al, 2013).

Um diagnóstico positivo para o HIV pode gerar um grave impacto na vida das mulheres, especialmente quando o diagnóstico ocorre durante o período gestacional, pois a maternidade se revela como sinal de vida e esperança em contraposição à ideia de morte relacionada à AIDS (ARAÚJO et al, 2008).

Apesar da descoberta do HIV/AIDS, ter se dado em meados dos anos 80, alguns detalhes importantes sobre a doença ainda são desconhecidos por muitas pessoas. Isso propicia em certo grau, o surgimento de preconceito e infelizmente, comportamentos discriminatórios por parte da sociedade, e até mesmo afetando na prática dos profissionais de saúde para com portadores de HIV (SCHERER et al, 2009).

Esse tipo de posicionamento frente à falta de informação ou esclarecimento adequado e suficiente sobre a doença afeta tanto a mulher gestante soropositiva que se vê frente a uma nova e desafiadora realidade, como aos profissionais da saúde que precisam prestar os cuidados incumbidos a eles a essas mulheres. Muitas vezes, a falta de consciência da importância do desempenho de uma 
assistência de qualidade, influencia até mesmo na adesão da gestante soropositiva ao tratamento a ela proposto durante o pré-natal (SILVA et al, 2008).

Entendemos que a interação terapêutica entre enfermeiros e gestantes HIV positiva durante o pré-natal, reforça a importância da adesão à profilaxia instituída pelo Ministério da Saúde com o intuito de reduzir os números de transmissão vertical.

Em geral, para as mulheres, a gravidez é idealizada como uma experiência única e complexa, intercalando vivências ambivalentes, como segurança/insegurança, alegria/tristeza, amor/raiva. No caso das gestantes soropositivas, há ainda um diferencial, pois além dos inúmeros sentimentos que cercam a mãe durante a gestação, precisa lidar ainda com a ansiedade, culpa e medo pela probabilidade de contaminação do filho pela transmissão vertical. (TEIXEIRA et al, 2013).

Apesar de o esquema profilático da terapia antirretroviral diminuir consideravelmente o risco de infecção, trazendo certa tranquilidade para a gestante, ainda permanece a condição de ansiedade, pois algumas informações dadas durante 0 aconselhamento sobre terapia antirretroviral geram expectativas negativas, ou seja, a terapia diminui significativamente o risco de infecção, porém ele ainda existe (CARTAXO et al, 2013).

Muitos enfermeiros ainda sentem certo impacto quando se veem frente a um paciente soropositivo. Quando além de soropositivo, se veem frente a uma mulher gestante soropositiva, os impactos torna-se ainda maior, devido ao estigma de ser uma doença altamente letal e acarretar tantos malefícios, tanto para a mãe quanto para o bebê se a gestante não receber uma assistência pré-natal adequada. Sejam por falta de preparo ou conhecimento, muitos profissionais chegam a desencorajar mulheres soropositivas a engravidarem, assumindo assim, um papel que não lhes pertence (CALDAS et al, 2015).

O papel do enfermeiro não é desencorajar a gestação, mas ajudar a mulher soropositiva na tomada de decisões, com pleno conhecimento a respeito da sua saúde reprodutiva, minimizando a probabilidade de transmissão vertical do HIV. Além disso, propiciar um vínculo terapêutico entre profissionais e gestantes de forma que não sejam apenas reconhecidas as responsabilidades de cada um neste processo, mas também de todos que estão envolvidos (direta ou indiretamente) no 
tratamento da mãe e do bebê e na prevenção da transmissão vertical do HIV (CECHIM et al, 2007).

Diante dessa situação, a relevância acadêmica do estudo se dá em reconhecer a importância do enfermeiro no acompanhamento de gestantes HIV positivas com relação à diminuição da ocorrência da transmissão vertical do vírus, através de aconselhamento e educação em saúde durante o pré-natal, pois essas mulheres encaram muitas dificuldades tanto em seu meio familiar quanto no social, o que acaba dificultando a adesão às recomendações para a profilaxia da transmissão vertical.

Com o objetivo de diminuir a taxa da transmissão do HIV de mãe para filho, há uma série de intervenções preconizadas pelo Ministério da Saúde. Dentre elas há a recomendação de um conjunto de medidas a serem feitas durante o pré-natal, parto e puerpério de mulheres portadoras do vírus HIV. Quando tais medidas são realizadas de maneira eficaz o risco da transmissão vertical reduz para 1 a $2 \%$ (ZIMMERMMANN et al, 2011).

Dentre as medidas, encontram-se: o uso de antirretrovirais a partir da $14^{\mathrm{a}}$ semana de gestação; a utilização de zidovudina injetável durante o trabalho de parto; a realização de parto cesáreo, quando indicado; o uso de antirretroviral oral para os recém-nascidos expostos, do nascimento até às 4 semanas de vida e inibição de lactação associada ao fornecimento de fórmula infantil até os seis meses de idade (BRASIL, 2014).

Dentre os elementos que colaboram para o aumento do risco da transmissão vertical do HIV, está o tempo entre a ruptura das membranas amnióticas e o parto, a quantidade de vírus nas secreções cérvico-vaginais e a quantidade de vírus no leite materno (TEIXEIRA et al, 2013). 


\section{PROBLEMA}

As orientações e o acompanhamento do enfermeiro são importantes para minimizar a probabilidade de transmissão vertical do HIV?

\section{PRESSUPOSTOS}

O acompanhamento do enfermeiro e suas orientações as gestantes HIV positivas é de grande relevância, visto que interfere na diminuição da ocorrência da transmissão vertical do vírus, através de aconselhamento e educação em saúde durante o pré-natal.

\section{OBJETIVOS}

\subsection{OBJETIVO GERAL}

Verificar a assistência do enfermeiro na prevenção da transmissão vertical do HIV.

\subsection{OBJETIVOS ESPECÍFICOS}

- Destacar as medidas profiláticas para prevenção da transmissão vertical do HIV;

- Descrever o papel do enfermeiro na assistência a gestante e prevenção. 


\section{JUSTIFICATIVA E RELEVÂNCIA:}

A motivação do estudo se deu a partir de pesquisas sobre o tema onde constatamos a dificuldade dos enfermeiros na assistência à gestante soropositiva e pelo fato de em alguns casos, a mulher não aderir totalmente ao tratamento.

No Brasil, Segundo a Secretaria de vigilância em saúde (MS) no período de 2000 até junho de 2016, foram notificadas 99.804 gestantes infectadas pelo HIV. Verificou-se que $39,8 \%$ das gestantes residiam na região Sudeste, seguida pelas regiões Sul (30,8\%), Nordeste (16,2\%), Norte $(7,4 \%)$ e Centro-Oeste $(5,7 \%)$. Em 2015, foram identificadas 7.901 gestantes no Brasil, sendo $31,9 \%$ na região Sudeste, $29,6 \%$ no Sul, $20,9 \%$ no Nordeste, $11,8 \%$ no Norte e $5,8 \%$ no CentroOeste.

A taxa de detecção de gestantes com HIV no Brasil vem apresentando tendência de aumento nos últimos dez anos; em 2006, a taxa observada foi de 2,1 casos/1.000 nascidos vivos, a qual passou para $2,7 \mathrm{em} \mathrm{2015}$, indicando um aumento de $28,6 \%$. A tendência de crescimento também é observada em todas as regiões do Brasil, exceto na região Sudeste, que permaneceu estável, com taxa de 2,2 casos/1.000 nascidos vivos em 2006 e 2,1 em 2015.

Desde 2000, a faixa etária de 20 a 24 anos é a que apresenta o maior número de casos de gestantes infectadas com HIV $(28,6 \%)$, notificadas no SINAN. Segundo a escolaridade, observa-se que a maioria das gestantes infectadas com HIV possui da $5^{\text {a }}$ à $8^{\text {a }}$ série incompleta, representando $30,1 \%$ dos casos notificados em 2015.

Diante disso este estudo tem como relevância social conscientizar as mulheres portadoras de HIV quanto à importância à adesão ao tratamento durante a gestação e no decorrer do pré-natal, assim aumentando a possibilidade de gerar um filho HIV negativo.

Para que essas ações tragam resultados desejáveis, se faz necessário que todos os profissionais envolvidos façam dessas questões uma realidade. Sendo assim, o profissional de enfermagem que tem em sua essência formadora o cuidado, assume uma responsabilidade para agir como educadores para com a saúde, desenvolvendo um trabalho de promoção e proteção ao ser humano. 


\section{MÉTODO}

Trata-se de uma Revisão integrativa realizada a partir de levantamento bibliográfico eletrônico em todas as bases de dados contidas na Biblioteca Virtual em Saúde (BVS): LILACS (Literatura Latino-Americana e do Caribe em Ciências da Saúde), IBECS (Índice Bibliográfico Espanhol de Ciências da Saúde), MEDLINE (Literatura Internacional em Ciências da Saúde), Biblioteca Cochrane e SCIELO (Scientific Electronic Library Online).

Os tesauros (descritores) foram determinados a partir das ferramentas DeCS (Descritores em Ciências da Saúde), do Portal BVS: transmissão vertical de doenças infecciosas; HIV; enfermagem. Foi utilizado o operador booleano AND.

Critérios de inclusão: Todos os tipos de estudos (experimentais, quase experimentais, observacionais e de revisão) que tratassem da transmissão vertical do HIV, além do papel do enfermeiro nestas comorbidades; em português; publicados entre os anos de 2012 a 2017. Critérios de exclusão: artigos de reflexão; revisões sem metodologia clara e reproduzível; estudos contendo apenas registros de ensaios clínicos e/ou resumos de revisões integrativas; estudos com populações não humanas. 


\section{RESULTADOS E DISCUSSÃO}

A pesquisa foi realizada entre agosto de 2017 e fevereiro de 2018. Os trabalhos foram lidos com o intuito de averiguar se preencheriam os critérios de inclusão por dois revisores, de forma independente. Foram cruzados os descritores transmissão vertical de doenças infecciosas, HIV e enfermagem e obtiveram-se 11 artigos, após colocação dos filtros, texto completo, idioma e recorte temporal, nenhum artigo foi encontrado.

Após, cruzou-se os descritores transmissão vertical de doenças infecciosa e HIV, obteve-se 1.021 artigos, após colocação dos filtros, texto completo, idioma e recorte temporal, obteve-se 8 artigos, os quais, após leitura de título e resumo, selecionou-se 3 artigos.

QUADRO 1: Título do artigo, autor (es), ano e objetivo principal

\begin{tabular}{|c|c|c|c|}
\hline Título do artigo & Autor (es) & Ano & Objetivo principal \\
\hline $\begin{array}{l}\text { Uso de drogas llícitas } \\
\text { por gestantes } \\
\text { Infectadas pelo HIV. }\end{array}$ & $\begin{array}{l}\text { Melo, Victor Hugo } \\
\text { Botelho, Ana Paula Machado } \\
\text { Maia, Marcelle Marie Martins } \\
\text { Júnior, Mário Dias Correa } \\
\text { Pinto, Jorge Andrade } \\
\end{array}$ & 2014 & $\begin{array}{l}\text { Determinar se o uso } \\
\text { de drogas ilícitas } \\
\text { aumenta a transmissão } \\
\text { vertical do HIV. }\end{array}$ \\
\hline $\begin{array}{l}\text { Prevalência de } \\
\text { infecções congênitas e } \\
\text { perinatais em } \\
\text { gestantes HIV positivas } \\
\text { da } \\
\text { região metropolitana de } \\
\text { Belo Horizonte. }\end{array}$ & $\begin{array}{l}\text { Maia, Marcele Marie Martins } \\
\text { Lage, Eura Martins } \\
\text { Moreira, Bárbara Cecília } \\
\text { Elayne, Braga de Deus } \\
\text { Faria, Joanna Gonçalves } \\
\text { Pinto, Jorge Andrade } \\
\text { Melo, Victor Hugo }\end{array}$ & 2015 & $\begin{array}{l}\text { Avaliar a prevalência } \\
\text { de HIV, toxoplasmose, } \\
\text { rubéola, } \\
\text { citomegalovírus, } \\
\text { hepatites B e C e sífilis } \\
\text { (Torchs) em uma } \\
\text { coorte de gestantes, } \\
\text { bem como identificar os } \\
\text { fatores } \\
\text { sociodemográficos, } \\
\text { clínicos e laboratoriais }\end{array}$ \\
\hline $\begin{array}{l}\text { Soro prevalência para } \\
\text { toxoplasmose, sífilis, } \\
\text { hepatite b, hepatite c, } \\
\text { rubéola, } \\
\text { citomegalovírus e vírus } \\
\text { da imunodeficiência } \\
\text { humana em gestantes } \\
\text { atendidas no } \\
\text { hospital universitário } \\
\text { Antônio Pedro, Niterói } \\
\text { (RJ) entre } 2008 \text { e } 2012 \text {. }\end{array}$ & $\begin{array}{l}\text { Vilte, Remberto Mauricio de } \\
\text { la Cruz Vargas } \\
\text { Azevedo, Kátia Martins } \\
\text { Lopes } \\
\text { Setúba, Sérgio } \\
\text { Oliveira, Solange Artimos }\end{array}$ & 2016 & 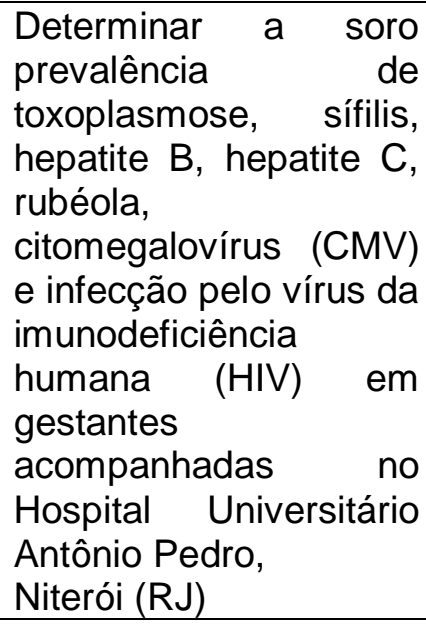 \\
\hline
\end{tabular}


QUADRO 2: Título do artigo e principais resultados.

\begin{tabular}{|c|c|}
\hline Título do Artigo & Principais Resultados \\
\hline $\begin{array}{l}\text { Uso de drogas llícitas por gestantes } \\
\text { Infectadas pelo HIV. }\end{array}$ & $\begin{array}{l}\text { O estudo revelou que o uso de drogas } \\
\text { ilícitas na gravidez entre mulheres } \\
\text { infectadas pelo HIV } \\
\text { é frequente. Assim, a abordagem sobre } \\
\text { o uso dessas drogas deve fazer parte da } \\
\text { rotina pré-natal. Essas gestantes são } \\
\text { mais } \\
\text { discriminadas, o que predispõe à } \\
\text { negação, à não procura ou ao acesso } \\
\text { tardio ao pré-natal }\end{array}$ \\
\hline $\begin{array}{l}\text { Prevalência de infecções congênitas e } \\
\text { perinatais em gestantes HIV positivas da } \\
\text { região metropolitana de Belo Horizonte. }\end{array}$ & $\begin{array}{l}\text { O estudo revelou que a prevalência das } \\
\text { Torchs se mostrou elevada para } \\
\text { algumas infecções. Conclui-se que é } \\
\text { importante manter o rastreamento de } \\
\text { Torchs na gravidez, especialmente nas } \\
\text { gestantes HIV positivas, para que se } \\
\text { possa estabelecer diagnóstico e } \\
\text { tratamento, e/ou medidas preventivas } \\
\text { para evitar a transmissão materno-fetal }\end{array}$ \\
\hline $\begin{array}{l}\text { Soro prevalência para toxoplasmose, } \\
\text { sífilis, hepatite b, hepatite c, rubéola, } \\
\text { citomegalovírus e vírus da } \\
\text { imunodeficiência humana em gestantes } \\
\text { atendidas no hospital universitário } \\
\text { Antônio Pedro, Niterói (RJ) entre } 2008 \text { e } \\
2012 \text {. }\end{array}$ & $\begin{array}{l}\text { Pode se verificar que o número de } \\
\text { gestantes susceptíveis à toxoplasmose } \\
(38,8 \%) \text { e ao vírus da hepatite } \mathrm{B}(\mathrm{VHB}) \\
(66,3 \%) \text { revela a necessidade de } \\
\text { medidas diagnósticas e preventivas da } \\
\text { toxoplasmose durante a gestação e } \\
\text { vacinação para o VHB, visando diminuir } \\
\text { o risco dessas infecções durante a } \\
\text { gravidez, melhorando, assim, a saúde } \\
\text { materno-infantil. }\end{array}$ \\
\hline
\end{tabular}


CATEGORIA 1. HIV (VÍRUS DA IMUNODEFICIÊNCIA HUMANA) E A TRANSMISSÃO VERTICAL

A AIDS é a manifestação clinica decorrente de um quadro causado pelo HIV. Esse vírus pertence à família Retroviridal, que causa efeitos patológicos na célula em curto prazo e uma infecção que alcança um quadro clínico geral. O crescimento de casos de AIDS entre mulheres teve, como consequência, o aumento da transmissão vertical da infecção pelo HIV, ou seja, a transmissão do vírus do HIV da mãe para o filho (a), durante a gestação, durante o trabalho de parto, no momento do parto, durante a amamentação, com elevação do número de casos de AIDS em crianças (LAZZAROTO, 2013).

Atualmente, 16 milhões de mulheres vivem com o HIV e muitas delas em idade reprodutiva. São complicadas pela infecção por HIV em todo o mundo 1.600.000 gestações e estima-se que as taxas de transmissão vertical (TV) sejam, na ausência de intervenção terapêutica, tão elevadas quanto $31 \%$. Entre as infecções em crianças menores de 15 anos, 90\% correm através de TV, e 90\% destas ocorrem na África Subsaariana.

Em 2011 ocorreram aproximadamente 330.000 novas infecções pediátricas, trazendo o número total de crianças infectadas mundialmente a assustadores 3,3 milhões desde o início da epidemia, com cerca de 1800 novas infecções diariamente sendo transmitidas de mães para seus bebês, através da gestação, parto ou amamentação. Entre as crianças infectadas, metade morre até os dois anos de vida na ausência de tratamento adequado. O diagnóstico precoce e o início imediato do tratamento podem reduzir a mortalidade e a progressão da doença em até $75 \%$ (SANTOS 2012).

O HIV é transmitido nos líquidos orgânicos que contêm vítrions livres e células T CD4+ infectadas. Esses líquidos incluem o sangue, o líquido seminal, as secreções vaginais, o líquido amniótico e o leite materno. A quantidade de HIV e de células infectadas no líquido orgânico está associada à probabilidade de que a exposição irá resultar em infecção. O HIV tem como alvo células com receptores CD4, sendo que aproximadamente $66 \%$ das células $T$ do sangue periférico consistem em células CD4+ (SMELTZER et al, 2012).

Segundo Costa (2013), à transmissão do vírus ao feto pode se dar de quatro formas distintas: por via transplacentária, por meio da secreção vaginal durante o parto, ingestão de fluídos maternos contaminados e através do leite materno. 
A transmissão mãe-filho da infecção pelo HIV constitui a principal via de contágio em crianças com idades inferiores há 15 anos, com mais de $90 \%$ dos casos de SIDA pediátrica ocorrendo por esta via. $\mathrm{Na}$ ausência de qualquer medida preventiva, as taxas de transmissão variam entre $15-30 \%$ nos países desenvolvidos, podendo atingir valores tão elevados como $30-45 \%$ nos países em vias de desenvolvimento. Esta diferença é principalmente atribuída às práticas de amamentação prolongada dos recém-nascidos nos países em vias de desenvolvimento (LAZZAROTO, 2013).

\section{CATEGORIA 2. INCIDÊNCIA DA TRANSMISSÃO VERTICAL}

Dados epidemiológicos mostram que, em relação à faixa etária, dos 25 aos 49 anos, tem-se $78 \%$ de homens infectados e $71 \%$ de mulheres, caracterizando a feminilização da epidemia. Devido a isso, as estratégias de prevenção destinadas às mulheres têm sido cada vez mais intensificadas (PEREIRA et al, 2012).

De acordo com o Ministério da Saúde, o risco de que a transmissão vertical ocorra, sem nenhum ato profilático é de $25 \%$ a $30 \%$. Se aplicadas todas as medidas preconizadas, a taxa de transmissão vertical do HIV seria reduzida para níveis inferiores a 2\% (BRASIL I, 2014). A taxa de detecção de casos de HIV em gestantes no Brasil em 2012 correspondeu a 2,4 casos por 1.000 nascidos vivos.

A única região com uma taxa de detecção superior à média nacional foi a Região Sul, com 5,8 casos por 1.000 nascidos vivos. No período de 2003 a 2012 , evidencia-se no Brasil um aumento de $26,3 \%$ na taxa de detecção de HIV em gestantes (HOFFMANN et al, 2014).

Em relação à categoria de exposição entre os indivíduos menores de 13 anos, a quase totalidade dos casos teve como via de infecção a transmissão vertical. Desde o início da epidemia de AIDS em 1980 até dezembro de 2013, foram constatados 278.306 óbitos tendo como causa fundamental a AIDS. Observando-se uma tendência significativa de queda nos últimos dez anos para o Brasil como um todo, o qual passou de 6,1 óbitos para cada 100 mil habitantes em 2004 para 5,7 em 2013, representando uma queda de $6,6 \%$. Sendo que em 2013, entre as 27 Unidades da Federação, 17 (63,0\%) apresentam coeficiente de mortalidade inferior à média nacional (BRASIL, 2014). 
De acordo com o Ministério da Saúde, de janeiro de 2011 a agosto de 2012, 21.162 crianças de 0 a 12 anos de idade foram acompanhadas nos serviços especializados de AIDS em todo Brasil, incluindo crianças expostas ao HIV e infectadas (BRASIL, 2015).

\section{CATEGORIA 3. DIAGNÓSTICO DA INFECÇÃO PELO HIV}

O diagnóstico da infecção pelo HIV no Brasil pode ser feito por meio da realização de ensaios denominados Elisa, imunofluorescência indireta, imunoblot, western blot e mais recentemente, a partir de julho de 2005, por meio da realização dos testes rápidos. Todos os laboratórios públicos, privados e conveniados ao Sistema Único de Saúde (SUS) devem seguir o disposto na Portaria 59/GM/MS, de 28 de janeiro de 2003 (HOFFMANN et al, 2014).

O conjunto de procedimentos sequenciados utilizados para a realização e conclusão do diagnóstico é denominado algoritmo ou fluxograma de testes. O diagnóstico da infecção pelo HIV utilizando-se testes rápidos pode ser feito em algumas maternidades selecionadas pelo Ministério da Saúde, são fundamentais para a indicação de quimioprofilaxia da transmissão vertical do HIV em gestantes no final do terceiro trimestre de gestação, em parturientes e em mulheres no pós-parto imediato, que não tenham sido testadas para o anti-HIV no pré-natal (SMELTZER et al, 2012).

Para isto, o MS capacita à equipe de profissionais de saúde envolvidos com esta atividade e fornece os insumos. A testagem rápida, portanto, não se constitui exame com finalidade de diagnóstico da mulher. Seu resultado deverá ser sempre considerado provisório, sendo imprescindível encaminhar a amostra reagente, ou a mulher, o mais rápido possível, após o parto, e em caráter prioritário, para a realização de testes confirmatórios (BRASIL, 2015).

\section{CATEGORIA 4. MEDIDAS PROFILÁTICAS PARA PREVENÇÃO DA TRANSMISSÃO VERTICAL DO HIV}

- Oferecer teste anti-HIV, com aconselhamento pré e pós-teste, para todas as gestantes nos serviços de pré-natal, devendo a testagem ser sempre voluntária e confidencial; 
- Ministrar nas gestantes HIV positivo o AZT oral (a partir da $14^{a}$ sem.); o AZT endovenoso nas parturientes, durante 0 trabalho de parto e parto até 0 clampeamento do cordão umbilical; e o AZT oral por seis semanas ao recémnascido (segundo diretrizes do Protocolo 076 do ACTG);

- Escolher a via de parto de acordo com os critérios de carga viral;

- Orientar a gestante/puérpera a substituição da amamentação com a introdução de leite artificial e outros alimentos, de acordo com a idade da criança, garantindo seu adequado crescimento e desenvolvimento.

A história natural da AIDS vem sendo alterada, consideravelmente, pela terapia anti-retroviral (ARV) que retarda a evolução da infecção, até seu estádio final, em que surgem as manifestações definidoras da Aids. Juntamente com as campanhas de prevenção, os ARV parecem estar contribuindo para a estabilização do crescimento da epidemia de AIDS no Brasil.

\section{CATEGORIA 5. PAPEL DO ENFERMEIRO NA ASSISTÊNCIA A GESTANTE E A PREVENÇÃO DA TRANSMISSÃO VERTICAL}

A assistência de enfermagem durante a gestação esta relacionada à consulta pré-natal, período no qual é realizado o acompanhamento da gestante até o momento do parto, tomando a evolução clinica tanto da mãe como do concepto, a fim de identificar riscos maternos e fetais (LIMA, PEREIRA, 2012).

$O$ enfermeiro necessita conhecer a patologia, consequências físicas e psicológicas associadas a AIDS, e dominar as habilidades do tratamento clínico para que assim preste uma assistência de qualidade a essa gestante portadora de HIV (SANTOS 2012).

O Ministério da Saúde recomenda a realização do teste anti-HIV, para todas as gestantes, na primeira consulta pré-natal Determina ainda, que a sorologia para HIV seja repetida, no início do terceiro trimestre, visando à saúde materno-infantil, com ênfase na prevenção da transmissão vertical para o recém-nascido. Quando a testagem não é realizada, durante o pré-natal, a mesma deve ocorrer, no momento do parto, por meio do teste rápido (PEREIRA et al, 2012).

Além das recomendações prestadas a gestante soropositiva, o enfermeiro também deve explicar sobre a importância da adesão ao tratamento com uso de anti-retrovirais ( a partir da $14^{\circ}$ semana de gestação) pois o mesmo é de total 
importância para proteção do seu filho e prevenção da transmissão vertical (SANTOS, 2012).

Para que o tratamento profilático tenha uma boa eficácia é imprescindível o acompanhamento do enfermeiro, pois o mesmo é responsável e participa ativamente por etapas muito importantes como o pré, peri e pós natal onde este é um momento ideal para que seja ofertado o teste anti-HIV e esclarecer dúvidas quanto à adesão dessa profilaxia (LANGENDORF, 2012). A assistência dada a mulher durante a gravidez não deve ser somente em relação à questão assistencial devendo também atender a gestante de forma humanizada, ou seja, além das intervenções clinicas deve promover uma relação interpessoal (CARTAXO et al, 2013).

Em estudo realizado com parturientes em 2010/2011, verificou-se que aproximadamente $99 \%$ das gestantes fizeram pelo menos uma consulta de prénatal, sendo que, dessas, 69,9\% fizeram seis consultas ou mais. A cobertura de testagem para HIV no pré- natal passou de 62,3\% em 2006 para 83,5\% em 2010, enquanto que a de sífilis (dois testes) passou de 16,9\% para 41,4\%, nesse mesmo período (BRASIL D, 2014).

Com a implantação da Rede Cegonha, em 2011, que objetiva certificar à mulher uma rede de cuidados e à criança o direito do nascimento seguro e ao crescimento e desenvolvimento saudáveis, houve considerável ampliação na oferta de testes rápidos de HIV e sífilis, o que implicou o aumento do diagnóstico ao longo dos anos no país (BRASIL D, 2014).

As gestantes apresentam reações frente a descoberta do diagnóstico positivo para o HIV. No primeiro momento, estas reações manifestam-se sob a forma de sentimentos de desespero, atribuindo à infecção um fator decisivo de sua sentença de morte, levando-as a analisar seus planos pessoais e familiares. Outro sentimento que emerge é o inconformismo e indignação, especialmente, para aquelas que não se consideravam como vulneráveis à infecção pelo vírus da AIDS (PEREIRA et al, 2012).

Para essas gestantes soropositivas essa é uma situação paradoxal, pois a gestação tem para todas as mulheres um significado muito forte e uma simbologia de amor, proteção e afeto. Uma redefinição no papel de mãe causa na mesma certa melancolia e uma sensação de falta de vinculo afetivo com o filho já que a mesma é 
orientada a não amamentação, ao parto cesáreo e o uso de vários comprimidos por dia (CARTAXO et al, 2013).

Muitas dessas gestantes se preparam para amamentar seu bebê, e esse direito the é negado. Embora com pouca ou nenhuma instrução formal, carregam consigo o conhecimento empírico sobre os benefícios do aleitamento materno (SCATTOLIN, JARDIM, 2012).

O leite agora passa a uma causa de morte e adoecimento perdendo seu significado de fonte de vida para seu bebê, o parto agora passa a ter mais um fator de risco, causando assim na mãe um misto de sensações. O fator que contribui para que a mãe adote o tratamento e siga as orientações para prevenção da transmissão vertical é o fato da mesma reconhecer e ter conhecimento que essas medidas servem como proteção para seu filho, assim as gestantes sentem-se na obrigação em aderir ao tratamento causando um estado de apreensão, tristeza e culpa (CARTAXO et al, 2013).

Portanto, além de todo o contexto da patologia, o enfermeiro deve proporcionar uma assistência humanizada a essa gestante soropositiva juntamente com um acompanhamento do profissional psicólogo para que os efeitos emocionais por conta desse diagnóstico sejam minimizados. 


\section{CONCLUSÃO}

A responsabilidade do enfermeiro tem grande relevância nesse processo de prevenção da transmissão vertical e também de orientação à essa mulher infectada, pois o mesmo além de detectar o HIV precocemente irá orientar a mesma a tomar os devidos cuidados, bem como fazer essa mãe conhecer um pouco mais sobre sua patologia.

Sendo assim, é evidente a importância da assistência de enfermagem na prevenção da transmissão vertical, principalmente pelo fato de obter um diagnóstico precoce do HIV, para que as taxas de transmissão vertical reduzam, ao contrário do que vem acontecendo no Brasil, aonde as taxas vêm aumentando gradativamente a cada ano.

Almeja-se através deste estudo, proporcionar às equipes de enfermagem e acadêmicos, um ganho de informações que levem a uma visão no que diz respeito à prevenção da transmissão vertical, com o propósito de prestar uma assistência completa e o mais humanizado possível. 


\section{REFERÊNCIAS}

1. ARAÚJO, Breno Fauth de. Mãe HIV positivo. In: NADER, Silvana Salgado; ARAÚJO, Breno Fauth de. Cuidado Integral do Recém Nascido: Prevenção e Condutas Terapêuticas. Rio de Janeiro: Rubio, 2015.

2. ARAÚJO, C.L.F; SIGNES, AF; ZAMPIER, V.S.B. O cuidado à puérpera com HIV/AIDS no alojamento conjunto: A visão da equipe de enfermagem. Esc. Anna Nery. Rio de Janeiro, v. 16, n. 1, p. 49-56, 2012. Disponível em: http://www.scielo.br/scielo.php?script=sci_arttext\&pid=S1414-81452012000100007. Acesso em 28 fev. 2018.

3. ARAÚJO, C.L.F.; AGUIAR, P.S.; SANTOS, G.K.A.; OLIVEIRA, M.G.P.; CÂMARA, L.S. A testagem anti-HIV nos serviços de ginecologia do município do Rio de Janeiro. Escola Anna Nery Revista de Enfermagem, v.18, n.1, p. 82-89, 2014. Disponível em: http://www.scielo.br/scielo.php?pid=S1414$81452014000100082 \&$ script=sci_abstract\&tlng=pt. Acesso em 18 fev. 2018.

4. ARAÚJO, M.A.L.; QUEIROZ, F.P.A.; MELO, S.P.; SILVEIRA, C.B.; SILVA, R.M. Gestante portadora do HIV: enfrentamento e percepção de uma nova realidade. Cienc. Cuid. Saúde, p. 216-223, Abr/Jun, 2008.

5. A. L. Maria; B. S Cláudia, et al. Vivências de gestantes e puérperas com o diagnóstico do HIV. Rev. Bras. Enferm. Brasília 2008. Disponível em: http://www.scielo.br/scielo.php?script=sci_arttext\&pid=S0034-71672008000500010 Acesso em: 02 out. 2017.

6. BRASIL. Ministério da Saúde. Departamento de DST, AIDS e hepatites virais. O que é sistema imunológico. 2015, Brasília, DF. Disponível em:< http://www.aids.gov.br/pagina/o-que-e-sistema-imunologicos. Acesso em: 02 out. 2017.

7. BRASIL. Ministério da Saúde. Transmissão vertical do HIV e Sífilis: estratégias para redução e eliminação. 2014, Brasília, DF. Disponível em:< 
http://www.aids.gov.br/pagina/transmissãoverticaldohivesifilis>. Acesso em: 02 out. 2017.

8. CALDAS, M.A.G.; PORANGABA, S.C.F.; MELO, E.S.; GIR, E.; REIS, R.K.R. Percepção da equipe de enfermagem sobre a gravidez no contexto da infecção pelo HIV. Rev. Rene, São Paulo, v. 16, n. 1, p. 29-37, jan. 2015. Disponível em: http://www.redalyc.org/articulo.oa?id=324036185005. Acesso em 28 fev. 2018.

9. CARTAXO, C. M. B. et al. Gestantes portadoras de HIV/AIDS: aspectos psicológicos sobre a prevenção da transmissão vertical. Estudos de psicologia, v. 18, p. 3, 2013, Natal, RN.

10. CECHIM,P.L.; PERDOMINI, F.R.I.P. ; QUARESMA, L.M.. Gestantes HIV positivas e sua não adesão à profilaxia no pré-natal. Rev. Bras. Enferm. Brasília, v. 60, n. 5, p. 519-523, Out. 2007. Disponível em http://www.scielo.br/scielo.php?pid=S003471672007000500007\&script=sci_abstract\&tlng=pt. Acesso em 28 fev. 2018.

11. COSTA, M. C. O. et al. HIV/AIDS e Sífilis entre gestantes adolescentes e adultas jovens: fatores de exposição e risco dos atendimentos de um programa de DST/HIV/AIDS na rede pública de saúde/SUS, Bahia, Brasil. In: Revista Baiana de Saúde Pública, 35, 2011, Salvador, BA. Disponível em: http://www.scielo.br/scielo.php?script=sci_arttext\&pid=S2416-71672008000500010 Acesso em: 03 out. 2017.

12. HOFFMANN, I. et al. Fatores que interferem na Transmissão vertical do HIV: Revisão Integrativa. Enfermagem Obstétrica, v. 1, p. 1, 2014, Rio de Janeiro. Disponível em: http://www.scielo.br/scielo.php?script=sci_arttext\&pid=S00347167200123456987456 Acesso em: 03 out. 2017.3

13. LAZZAROTTO, A.; DERESZ, L.; SPRINZ, E. HIV/AIDS e treinamento concorrente: a Revisão Sistemática. Revista Brasileira de Medicina do Esporte, v. 16, p. 2, 2010, Niterói, RJ. Disponível em: http://www.scielo.br/pdf/rbgo/v37n9/0100-7203-rbgo-3907-00421.pdf. Acesso em 02 out. 2017. 
14.LIMA, A.C.M.A.C.C.; COSTA, C.C.; TELES, L.M.R.; DAMASCENO, A.K.C.; ORIÁ, M.O.B. Avaliação epidemiológica da prevenção da transmissão vertical do HIV. Acta Paul. Enferm., São Paulo, v. 27, n. 4, p. 311-318, ago. 2014. Disponível em: http://www.scielo.br/pdf/ape/v27n4/1982-0194-ape-027-004-0311.pdf. Acesso em 28 fev. 2018.

15. MAIA M. M. I. Maria; MARTINS M. Eura, et al. Prevalência de infecções congênitas e perinatais em gestantes HIV positivas da região metropolitana de Belo Horizonte. Rev. Bras. Ginecol. Obstet. 2015; v. 37. n. 9, p. 421-7. Disponível em: http://www.scielo.br/pdf/rbgo/v31n8/0120-7273-rbgo-37-09-004231.pdf. Acesso em 02 out. 2017.

16. MELO B. Victor Hugo; MAIA. M. Ana Paula, et al. Uso de drogas llícitas por gestantes Infectadas pelo HIV. Rev. Bras. Ginecol. Obstet. 2014; v. 36, n. 12, p. 55561. Disponível em: http://www.scielo.br/pdf/rbgo/v36n12/0100-7203-rbgo-36-120555.pdf. Acesso em 02 out. 2017.

17.PEREIRA, F.W. et al. Atendimento de gestantes em centro de testagem e aconselhamento na perspectiva dos profissionais. Revista de Enfermagem da UFSM, v. 2, p.2, 2012, Santa Maria, RS. Disponível em: http://www.scielo.br/pdf/rbgo/v37n14/4580-7203-rbgo-33-12-04555.pdf. Acesso em 02 out. 2017.

18.SANTOS, E.F.; OKAZAKI, E.L.F.J. Assistência de enfermagem à gestante soropositiva para HIV. Revista de Enfermagem UNISA, v. 13, p. 1, 2012, São Paulo, SP. Disponível em: http://ojs.cesuca.edu.br/index.php/revistaenfermagem/article/view/1228. Acesso em: 03 out. 2017.

19.SCATTOLIN, F.; JARDIM, V. M. R. Mães HIV positivo e a orientação para não amamentar: um relato de experiência. Journal of Nursing and Health, v.2, 2012. Disponível em: http://www.scielo.br/pdf/\%0D/rbsmi/v6n2/30917.pdf. Acesso em 03 out. 2017. 
20.SCHERER, L.M.; BORENSTEIN, M.S.; PADILHA, M.I. Gestantes/puérperas com HIV/AIDS: conhecendo os déficits e os fatores que contribuem no engajamento para o autocuidado. Esc. Anna Nery Rev. Enferm, Rio de Janeiro, v.13, n. 2, p. 358-365, jun. $2009 . \quad$ Disponível em: http://www.scielo.br/scielo.php?script=sci_abstract\&pid=S1414$81452009000200017 \&$ Ing=pt\&nrm=iso\&tIng=en. Acesso em 28 fev. 2018.

21.SILVA, R.M.O.; ARAÚJO, C.L.F.; PAZ, F.M.T. A realização do teste anti-HIV no prénatal: os significados para a gestante. Esc. Anna Nery Rev. Enferm, Rio de Janeiro, v. $12, \quad$ n. 4 , p. 630-636, dez. 2008. Disponível em: http://www.scielo.br/scielo.php?script=sci_arttext\&pid=S1414-81452008000400004. Acesso em 26 fev. 2018.

22. SMELTZER, S. C. et al. Brunner \& Suddarth: Tratado de Enfermagem MédicoCirúrgica. 12, v. 3. Editora Guanabara Koogan, 2012. p 1577.

23.SOARES, M.L.; OLIVEIRA, M.I.C.; FONSECA, V.M.; BRITO, A.S.; SILVA, K.S. Preditores do desconhecimento do status sorológico de HIV entre puérperas submetidas ao teste rápido anti-HIV na internação para o parto. Ciênc. Saúde Coletiva, Rio de Janeiro, v. 18, n.5, p.1313-1320, maio 2013. Disponível em: https://www.scielosp.org/article/ssm/content/raw/?resource_ssm_path=/media/assets /csc/v18n5/16.pdf Acesso em 26 fev. 2018.

24. TEIXEIRA, S. V. B.; SILVA, G. S.; SILVA, C. S.; MOURA, M. A. V. Mulheres soropositivas ao HIV: a decisão de engravidar. Revista de Pesquisa: cuidado é fundamental online, Rio de Janeiro: janeiro/março, ano 5 , no 1 p. 3159 - 3167, 2013. Disponível em: http://bases.bireme.br/cgibin/wxislind.exe/iah/online/?IsisScript=iah/iah.xis\&src=google\&base=LILACS\&lang=p \&nextAction=Ink\&exprSearch=686274\&indexSearch=ID. Acesso em 24 fev. 2018.

25. VILTE R. M. Vargas; AZEVEDO, M. L. S. Kátia, et al. Soro prevalência para toxoplasmose, sífilis, hepatite $\mathrm{b}$, hepatite $\mathrm{c}$, rubéola, citomegalovírus e vírus da imunodeficiência humana em gestantes atendidas no hospital universitário Antônio 
Pedro, Niterói (RJ) entre 2008 e 2012. Rev. Bras. Ginecol. Obstet. 2013; v. 35, n. 2, p. 66-70. Disponível em: http://www.scielo.br/pdf/rbgo/v35n2/05.pdf. Acesso em 03 out. 2017.

26.ZIMMERMMANN, J.B.; NEVES, H.S.; SOUZA, P.B.; PENA, D.M.F.; PEREIRA, M.P.; NUNES, T.R.; OLIVEIRA, P.L. Rastreio da infecção pelo vírus da imunodeficiência humana no momento do parto. Rev. Bras. Ginecol. Obstet. Minas Gerais, v. 33, n. 4, p. 170-175, abril 2011. Disponível em: http://www.scielo.br/scielo.php?script=sci_abstract\&pid=S0100$72032011000400004 \&$ Ing=en\&nrm=iso\&tlng=pt. Acesso em 24 fev. 2018. 